\title{
Diferença motivacional entre praticantes iniciantes e veteranos do taekwondo
}

\author{
Motivational diferences between beginners and veterans in taekwondo \\ DOI: http://dx.doi.org/10.36453/2318-5104.2018.v16.n1.p41
}

\author{
Vitor Bento Crozeta Barbosa ${ }^{1}$, Luana Caroline Contessoto ${ }^{1}$, Ana Luiza Barbosa Anversa ${ }^{1,2}$, \\ Luciana Boligon Refundini ${ }^{1}$ \\ ${ }^{1}$ Centro Universitário Metropolitano de Maringá (UNIFAMMA) \\ ${ }^{2}$ Universidade Estadual de Maringá (UEM)
}

\section{RESUMO}

O Taekwondo é uma arte marcial coreana baseada no respeito e disciplina. Como prática esportiva, a modalidade envolve diferentes fatores motivacionais o que acaba por direcionar o foco e objetivo de cada atleta durante a prática. Deste modo o objetivo desta pesquisa foi analisar as diferenças motivacionais de praticantes iniciantes e veteranos do Taekwondo. Participaram da pesquisa 24 atletas, de um centro de treinamento de Maringá-PR, com idade entre 18 e 31 anos, sendo que 12 dos participantes eram faixas brancas e 12 faixas pretas. Como instrumento de coleta utilizou-se a Escala de Motivação para E'sporte II (SMS II). Os dados foram analisados por meio do programa estatístico SPSS 15.0. Para comparar as categorias do teste de motivação em relação a graduação do atleta, utilizou-se o teste de Kruskal-Wallis e para ver as múltiplas comparações, o teste de Tukey. Os resultados demonstram que entre os praticantes iniciantes e veteranos a motivação intrínseca $(\mathrm{md}=6,00)$ e motivação extrínseca indentificada $(\mathrm{md}=6,00)$ foram as que apresentaram maiores índices, o que demonstra que o interesse pela prática é espontâneo ou apresenta alguma interiorização. Com relação à comparação entre iniciantes e veteranos, o único fator que apresentou diferença significativa $(p=0,000)$ foi à desmotivação em que os praticantes de faixas brancas apresentaramse menos desmotivados para os treinos do que os de faixa preta. Conclui-se que, dentre os dois grupos analisados, os níveis de motivação intrínseca e extrínseca, mostram-se igualados, porém, o nível de desmotivação de atletas faixas pretas encontra-se superior aos dos atletas faixas brancas iniciantes no Taekwondo. Estes dados demonstram que com o passar dos anos de treino a desmotivação pode vir a aumentar levando a evasão do esporte.

PALAVRAS-CHAVE: Motivação; Artes Marciais; Atletas.

\section{ABSTRACT}

Taekwondo is a korean martial art, based on respect and discipline. As a sports practice, is a modality that involves different motivational factors or it has just directed the focus and goal of each athlete during practice. In this way the objective of this research was to analyze the motivational differences between beginners and veterans in Taekwondo. Twenty-four athletes participated in the study, from a training center in Maringá (PR, Brazil)), aged between 18 and 31 years, of which 12 were white bands and 12 black bands. The Sports Motivation Scale II (SMS II) was used. The data were analyzed by means of the statistical program SPSS 15.0. In order to compare the categories of the motivation test in relation to the athlete's graduation, the Kruskal-Wallis test was used and to see as multiple comparisons, Tukey's test. The results show that among beginners and veterans, intrinsic motivation ( $\mathrm{md}=6.00$ ) and extrinsic motivation identified ( $\mathrm{md}=6.00)$, as we present higher indices, which make up the interest for the practice is spontaneous or presents interiorization. Regarding the comparison between beginners and veterans, the only factor that presented the significant difference $(p=0.000)$ for the demotivation which white bands were less motivated for training than the black belt. It is concluded that, among the two groups analyzed, the levels of intrinsic and extrinsic motivation, in general, are shown to be equal, however, the dismantling process of athletes black bands is superior to those of athletes white bands beginners in Taekwondo. These data demonstrate that over the years of training, demotivation may increase, leading to an avoidance of the sport.

KEYWORDS: Motivation; Martial Arts; Athletes. 


\section{INTRODUÇÃO}

As lutas ou formas de combate sempre fizeram parte dos costumes e manifestações corporais da humanidade. Para Mazzoni e Oliveira Júnior (s/d), desde os primórdios até os dias atuais o homem utiliza-se das manifestações corporais, como as lutas, para diferentes objetivos e situações tais como autodefesa ou mesmo para o condicionamento físico. Em seu viés competitivo, Rufino (2017) coloca que a prática evidencia o confronto direto entre os praticantes, pautando-se em regras e tendo como objetivo o desenvolvimento de ações de caráter simultâneo e imprevisível.

Existem as lutas que são classificadas como artes marciais, as quais estão apresentando um grande crescimento na sociedade atual. As artes marciais se caracterizam como atividades que, em sua maioria, respondem como modalidade esportiva. Não são poucos os torneios, campeonatos e patrocinadores dispostos a oferecer distintas formas de capitar às "novas promessas" do Jiu-Jitsu, Judô, Caratê e Taekwondo (PIMENTA; MARCHI JÚNIOR, 2009).

O Taekwondo é uma arte marcial coreana criada para defender fronteiras de cidades em conflitos fortalecendo o exército de cada nação, sendo baseada na cultura oriental tendo como principais elementos o respeito e a disciplina. Após a popularização desta arte marcial nas guerras, ela foi reconhecida como esporte, e mais tarde foi implantada nas Olimpíadas, sendo criadas assim suas regras oficiais (CHEMELLO; BONONE, 2014).

Como na maioria das artes marciais, o Taekwondo também é representado por faixas de diversas cores. As cores das graduações podem variar de acordo com determinadas regiões, com exceção da faixa branca que é sempre a inicial e da faixa preta que é a última das cores/graduação e são conquistadas por meio de exames avaliativos. Segundo Weiss e Chaumeton apud Pinto e Costa (2015), os praticantes de Taekwondo progridem por meio de uma estrutura de graduações pré-definidas, podendo desenvolver a partir destas, diferentes orientações motivacionais.

Ao longo do processo de treinamento e graduação de faixa, os professores e mestres de Taekwondo tem um papel muito importante no incentivo motivacional de seus alunos, principalmente, quando se trata de dar continuidade ao esporte e/ou participar de competições. Este incentivo emerge de fatores intrínsecos e extrínsecos ao praticante, que podem surgir do suporte à autonomia e dos trabalhos direcionados a percepção subjetiva de esforço, adequando o treino às condições do atleta e as aspirações de cada um (MARTINS; ROSADO, 2017).

A motivação é o processo que leva as pessoas a uma ação ou à inércia em diversas situações, sendo ainda o exame das razões pelas quais se escolhe fazer algo, executar algumas tarefas com maior empenho do que outras ou persistir numa atividade por longo período de tempo. Tresca e Rose Júnior (2000) afirmam que as teorias motivacionais destacam que um indivíduo pode ter como fonte de suas ações, tanto razões internas quanto externas.

De acordo com Samulski (2002) a motivação intrínseca está relacionada com a motivação que vem do interior do indivíduo e está relacionada, principalmente, ao prazer. Já, a motivação extrínseca está relacionada a fatores externos ao indivíduo, geralmente relacionados à obrigação de fazer algo, em vista de alguma recompensa. A desmotivação influência negativamente atitudes e respostas comportamentais dos indivíduos em praticamente todas as suas situações de vida, o que pode levar a um estado de frustração e desmotivação (RAGAZINI, 2011).

Durante a realização de uma prática esportiva ou no ambiente de competição, a motivação está relacionada não apenas com um bom desempenho, mas, também com a jornada psicológica para alcançálo (WEINBERG; GOULD, 2008). Desta forma, com base na teoria da autodeterminação os eventos sócios contextuais podem vir a fortalecer o nível de percepção de competência no decorrer do tempo, por exemplo, um feedback positivo em uma situação de desafio, pode vir a aumentar o nível da motivação intrínseca do indivíduo (GUIMARÃES; BORUCHOVITCH, 2004). Posto isso, o presente estudo objetivou analisar as diferenças motivacionais entre praticantes de Taekwondo iniciantes e veteranos. 


\section{MÉTODOS}

Participaram da pesquisa 24 alunos de Taekwondo de uma academia de Maringá (PR), com idades entre 18 a 30 anos, de ambos os sexos. 0 tempo de prática da amostra da modalidade é de 8,75 anos ( $\pm 4,32$ anos). Dentre os praticantes que compuseram a amostra, 12 são faixas brancas e praticam a modalidade a 5,9 anos $( \pm 2,27$ anos) e 12 faixas pretas, com 11,25 anos ( $\pm 4,09$ anos) de prática.

Os critérios adotados para a inclusão dos participantes foram: (a) ser praticante de Taekwondo, faixa preta ou branca e, vinculados à academia participante da pesquisa; (b) já ter participado de pelo menos um campeonato ou competição e (c) aceitar participar da pesquisa por meio da assinatura do Termo de Consentimento Livre e Esclarecido (TCLE)

Para coleta de dados foram aplicados, no próprio local de treino antes ou após as sessões de treinamento, uma ficha de identificação de modo a traçar dados quanto ao sexo, idade, tempo de prática, categoria que pertence e resultados obtidos em campeonatos e o questionário Escala de Motivação para Esporte II (SMS II) adaptado e traduzido para língua portuguesa por Nascimento Júnior et al. (2014), para avaliar o continuum de motivação para o esporte e qual o seu tipo.

O questionário possui 18 questões de múltiplas escolhas, com escala tipo likert de sete pontos (não corresponde totalmente à corresponde completamente). As questões são divididas em seis escalas: a) regulação intrínseca; b) regulação integrada; c) regulação identificada; d) regulação introjetada; e) regulação externa; e f) desmotivação.

A análise dos dados se deu de forma descritiva e inferencial. Os dados numéricos foram submetidos ao teste de normalidade Shapiro-Wilk, apresentando distribuição não normal por isso, utilizou-se mediana e intervalo interquartil. Para comparar as categorias do teste de motivação entre as faixas pretas e brancas utilizou-se Kruskal-Wallis, e para ver as múltiplas comparações o teste de Tukey. O nível de significância adotado foi $p<0,05$. A análise estatística foi realizada mediante o pacote computadorizado Statistical Package for the Social Science (SPSS) versão 15.0. Por fim, destaca-se que a coleta de dados seguiu os procedimentos Éticos em Pesquisa estabelecidos pelo Conselho Nacional de Saúde.

\section{RESULTADOS E DISCUSSÃO}

Para analisar as diferenças motivacionais de praticantes do Taekwondo os respondentes foram separados de acordo com a faixa, sendo iniciantes (faixa branca) e veteranos (faixa preta). No primeiro momento, buscouse identificar o perfil motivacional do grupo de atletas praticantes do Taekwondo da amostra (24 atletas) selecionada (Tabela 1).

Tabela 1. Escalas de motivação de atletas de Taekwondo de Maringá.

\begin{tabular}{lcc} 
Variável Motivacional & Mediana & (Q1:Q3) \\
Intrínseca & 6,00 & $(5,00 ; 6,00)$ \\
\hline Extrínseca Integrada & 5,00 & $(4,83 ; 6,00)$ \\
\multicolumn{1}{c}{ Identificada } & 6,00 & $(6,00 ; 6,16)$ \\
Introjetada & 5,00 & $(4,50 ; 6,00)$ \\
Externa & 1,33 & $(1,00 ; 2,00)$ \\
\hline Desmotivação & 2,00 & $(1,00 ; 2,66)$ \\
\hline
\end{tabular}

Os dados demonstram que na amostra a motivação intrínseca ( $m d=6,00$ ) e a motivação extrínseca identificada $(m d=6,00)$ apresentaram maiores índices em relação às demais fontes motivacionais, o que 
demonstra que o interesse pela prática é espontâneo ou apresenta alguma interiorização. De acordo com Deci e Ryan (1985), a regulação identificada representa uma forma autodeterminada dentro de um continuum de autonomia, o que demonstra que mesmo motivado por elementos externos o individuo valoriza conscientemente o objetivo inerente à determinada ação. Pedro e Martins (2017) apontam que quando o perfil motivacional de lutadores se enquadra nas motivações intrínseca, integrada e identificada os mesmos apresentam maior compromisso, esforço e persistência na prática. $\mathrm{O}$ que mostra que eles são bastante autodeterminados o que os ajuda a se manterem na prática.

Ao fazer a comparação dos scores de motivação entre os atletas em relação ao grau de graduação, faixa branca (iniciante) e faixa preta (veteranos) (Tabela 2), contatou-se que as medianas obtidas pelo grupo foram similares apresentando diferença significativa apenas na desmotivação $(p=0,000)$, em que os veteranos apresentaram índice de desmotivação $(m d=2,66)$ maiores do que os iniciantes ( $m d=1,00)$.

Tabela 2. Comparação das Medianas dos scores de motivação entre atletas faixa branca e atletas faixa preta de Taekwondo.

\begin{tabular}{|c|c|c|c|c|}
\hline Variável Motivacional & Faixa & Mediana & (Q1; Q3) & $\mathbf{p}$ \\
\hline \multirow{2}{*}{ Intrínseca } & Preta & 6,00 & $(5,66 ; 6,16)$ & \multirow{2}{*}{0,266} \\
\hline & Branca & 6,00 & $(5,00 ; 6,00)$ & \\
\hline \multirow{2}{*}{ Extrínseca Integrada } & Preta & 5,00 & $(4,83 ; 6,50)$ & \multirow{2}{*}{0,843} \\
\hline & Branca & 6,00 & $(4,50 ; 6,00)$ & \\
\hline \multirow{2}{*}{ Identificada } & Preta & 6,00 & $(6,00 ; 6,83)$ & \multirow{2}{*}{0,128} \\
\hline & Branca & 6,00 & $(5,50 ; 6,00)$ & \\
\hline \multirow{2}{*}{ Introjetada } & Preta & 5,00 & $(5,00 ; 6,00)$ & \multirow{2}{*}{0,319} \\
\hline & Branca & 5,00 & $(4,00 ; 5,50)$ & \\
\hline \multirow{2}{*}{ Externa } & Preta & 1,33 & $(1,00 ; 3,00)$ & \multirow{2}{*}{0,755} \\
\hline & Branca & 1,50 & $(1,00 ; 2,00)$ & \\
\hline \multirow{2}{*}{ Desmotivação } & Preta & 2,66 & $(2,00 ; 3,00)$ & \multirow{2}{*}{$0,000^{*}$} \\
\hline & Branca & 1,00 & $(1,00 ; 1,00)$ & \\
\hline
\end{tabular}

De acordo com a teoria da autodeterminação, o sentimento de desmotivação resulta no desenvolvimento da tarefa de forma desorganizada acompanhada de sentimento de frustração, medo ou depressão e passa a não esperar o resultado almejado (DECl; RYAN,1985). O resultado da desmotivação varia a cada praticante, podendo ser desde o alcance do objetivo do atleta ou a falta de uma meta a ser alcançada pelo mesmo. Segundo Samulski (2002), a motivação esportiva consiste num processo ativo, intencional e dirigido a uma meta, que depende da interação dos fatores pessoais e ambientais.

Com o estado de desmotivação o atleta pode não perceber motivos para se manter na atividade, o que pode levar a desistência da prática (FERNANDES; VASCONCELOS RAPOSO, 2005). Isso pode ter se dado com maior incidência em atletas veteranos, por já terem atingido o grau máximo de graduação no Taekwondo. Estes dados demonstram que com o passar dos anos de treino a desmotivação pode vir a aumentar levando a evasão do esporte, por isso cabe ao profissional responsável pelo treino e pelos atletas buscarem diferentes formas motivacionais para induzir benefícios cognitivos, comportamentais e afetivos que contribuam para a manutenção na prática.

Fernandes e Vasconcelos-Raposo (2005) destacam a importância de proporcionar percepções de tomada de decisão e de liderança, aumentando o envolvimento nas tarefas propostas, proporcionar feedbacks informacionais e não controladores e minimizar situações de comparação do rendimento esportivo. Desta forma, ao longo dos treinos de Taekwondo é importante que os atletas veteranos (faixa pretas) assumam 
posição de liderança, demonstrando movimentos ou atuando como tutores dos atletas iniciantes (faixa branca) e que mesmo com a técnica mais aprimorada, reflexo dos anos de treino e das etapas de graduação, continuem recebendo feedback de seus mestres, de modo a ter sentimento de aprovação/reconhecimento social.

\section{CONCLUSÃO}

Dentro do mundo esportivo, a questão motivacional está presente em todas as modalidades e é de extrema importância para o rendimento do aluno ou atleta, uma vez que o mesmo precisa de estímulos para se manter na carreira esportiva e lutar para obter o êxito a partir de suas vontades e metas. A partir dos resultados da pesquisa, constatou-se que os atletas de Taekwondo apresentaram valores mais altos na motivação intrínseca e motivações extrínsecas mais internas, enquanto tiveram valores mais baixos na desmotivação e nas motivações extrínsecas externas.

Quando comparados os atletas faixas brancas e pretas, os valores foram próximos, somente o fator desmotivação apresentou diferença significativa, sendo que os atletas faixas pretas encontram-se mais desmotivados do que os atletas iniciantes. Este fator pode vir a ocorrer por diversos fatores relacionados ao grupo ou, especificamente, as expectativas em relação a prática e seus resultados.

Por fim, ressalta-se que os trabalhos e pesquisas brasileiras relacionadas a modalidade de Taekwondo em questões motivacionais são bem escassos para a pesquisa e coleta de informações, assim novos estudos podem vir a melhorar a manutenção de atletas veteranos na modalidade e a intervenção do profissional responsável pelos treinos, além de complementar pesquisas científicas sobre a temática.

\section{REFERÊNCIAS}

CHEMELLO, G.; BONONE, C. G. G. Taekwondo - escola - família: comportamentos e atitudes de atletas da cidade de São Marcos-RS. Do Corpo: Ciências e Arte, Caxias do Sul, v. 4, n. 1, p. 1-16, 2014.

DECI, E. L.; RYAN, R. M. The general causality orientations scale: self-determination in personality. Journal of Research in Personality, Amsterdam, v. 19, n. 2, p. 109-34, 1985.

FERNANDES, H. M.; VASCONCELOS-RAPOSO, J. Continuum de autodeterminação: validade para a sua aplicação no contexto desportivo. Estudos de Psicologia, Campinas, v. 10, n. 3, p. 385-95, 2005.

GUIMARÃES, S. E. R.; BORUCHOVITCH, E. O estilo motivacional do professor e a motivação intrínseca dos estudantes: uma perspectiva da teoria da autodeterminação. Psicologia: Reflexão e Crítica, Porto Alegre, v. 17, n. 2, p. 143-50, 2004.

MARTINS, P.; ROSADO, A. A formação de treinadores de luta olímpica: estudos das fontes de conhecimento e conteúdos de formação essenciais. Revista de Artes Marciales Asiaticas, Lisboa, v. 12 , n. 1, p. 24-40, 2017.

MAZZONI, A. V.; OLIVEIRA JÚNIOR, J. L. Lutas: da pré-história à pós-modernidade. s/d. Disponível em: <http://www.gpef.fe.usp.br/teses/agenda_2011_04.pdf>. Acesso em: 01 de agosto de 2017.

NASCIMENTO JÚNIOR, J. R. A.; VISSOCI, J. R. N.; BALBIM, G. M.; MOREIRA, C. R.; PELLETIER, L.; VIEIRA, L. F. Adaptação transcultural e análise das propriedades psicométricas da Sport Motivation Scale-II no contexto brasileiro. Revista Educação Física/UEM, Maringá, v. 25, n. 3, p.441-58, 2014.

PEDRO, S.; MARTINS, P. Suporte de autonomia, empenhamento e percepçao subjetiva de esforço em lutadores. Revista Iberoamericana de Psicología del Ejercicio y el Deporte, Las Palmas, v. 12, n. 2, p. 279-86, 2017.

PIMENTA, T. F. F.; MARCHI JÚNIOR, W. A constituição de um subcampo do esporte: o caso do Taekwondo. Movimento, Porto Alegre, v. 15, n. 1, p. 193-215, 2009.

PINTO, S. A.; COSTA, G. C. T. Motivação dos praticantes de Taekwondo: Estudo realizado em academias de Belo Horizonte. Revista Brasileira de Futsal e Futebol, São Paulo, v. 7, n. 27, p. 495-504, 2015.

RAGAZINI, R. A motivação e a desmotivação: duas faces de uma mesma moeda na vida do trabalhador. 
2011. Disponível em: <http://www.administradores.com.br/artigos/carreira/a-motivacao-e-adesmotivacao-duas-faces-de-uma-mesma-moeda-na-vida-do-trabalhador/53311/>. Acessado em: 21 de março de 2017.

RUFINO, L. G. B. Lutas. In: GONZÁLEZ, F. J.; DARIDO, S.; OLIVEIRA, A. A. B. (Org.). Lutas, capoeira e práticas corporais de aventura. 2. ed. Maringá: Eduem, 2017.

SAMULSKI, D. M. Psicologia do esporte. Barueri: Manole, 2002.

TRESCA, R. P.; ROSE JÚNIOR, D. Estudo comparativo da motivação intrínseca em escolares praticantes e não praticantes de dança. Revista Brasileira de Ciência e Movimento, Brasília, v. 8, n. 1, p. 9-13, 2000.

WEINBERG, R. S.; GOULD, D. Fundamentos da psicologia do esporte e do exercício. 4. ed. Porto Alegre: Artmed, 2008.

Autor correspondente: Ana Luiza Barbosa Anversa

E-mail: ana.beah@gmail.com

Recebido: 26 de janeiro de 2018. Aceito: 23 de março de 2018. 